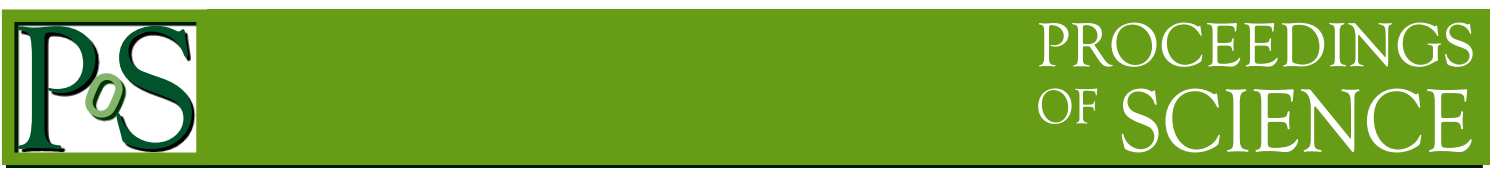

\title{
Mid-Decade Outlook for Balloon-Borne Particle Astrophysics Research
}

\author{
William Vernon Jones ${ }^{1}$ \\ NASA Headquarters, Science Mission Directorate, Astrophysics Division DHOOO \\ 300 E Street. SW, Washington DC 20546, USA \\ E-mail: w.vernon.jones@nasa.gov
}

The NASA Particle Astrophysics Program covers the Origin of the Elements, Nearest Sources of Cosmic Rays, How Cosmic Particle Accelerators Work, The Nature of Dark Matter, and Ultrahigh Energy Neutrinos. Progress in each of these topics has come from sophisticated instrumentation flown on Long Duration Balloon (LDB) flights around Antarctica for more than two decades. Super Pressure Balloons (SPB) and International Space Station (ISS) platforms are emerging opportunities that promise major steps forward for these and other objectives. NASA has continued development and qualification flights leading to SPB flights capable of supporting $1000 \mathrm{~kg}$ science instruments to $33 \mathrm{~km}$ for upwards of hundred day missions, with plans for increasing the altitude to $38 \mathrm{~km}$. This goal is even more important now, in view of the National Research Council Astro2010 Decadal Study recommendation that NASA should support Ultra-Long Duration Balloon (ULDB) flight development. Astro2010 emphasized that NASA should support such missions for indirect detection of dark matter and for cosmic-ray physics and astrophysics. Scientific ballooning as a vital infrastructure component for cosmic ray and general astrophysics investigations, including training for young scientists, graduate and undergraduate students, leading up to the 2020 Decadal Study and beyond, will be presented and discussed.

The 34th International Cosmic Ray Conference

30 July- 6 August 2015

The Hague, The Netherlands

${ }^{1}$ Speaker 


\section{Introduction}

The NASA particle astrophysics program has traditionally supported science investigations utilizing payloads flown on large stratospheric balloons that carry instruments above $99.5 \%$ of Earth's atmosphere, or similar-class payloads flown as flights of opportunity on space missions. The Astrophysics Division manages the Balloon Program, which utilizes large stratospheric balloons to carry science and technology instruments above about $99.5 \%$ of the Earth's atmosphere. It provides flight opportunities for investigations across the Science Mission Directorate (SMD), which includes the Divisions of Planetary Science, Heliophysics (which manages the Sounding Rocket Program) and Earth Science (which manages the Airborne Program). Annual Research Opportunities in Space and Earth Science (ROSES) solicitations allow prospective investigators to compete for funding needed to develop and fly their suborbital payloads.

Approximately 15-20 balloon experiments are launched each year from various locations: Ft. Sumner, NM; Kiruna, Sweden; Alice Springs, Australia: and McMurdo, Antarctica. Flight times range from several hours for launches in Fort Sumner, to several days for launches from Sweden to Canada, and from a couple of weeks to a couple of months for launches near McMurdo (Fig. 1) that circumnavigate Antarctica around the South Pole. The constant daylight means no day-night temperature fluctuations, so the balloon stays at a nearly constant altitude throughout the flight. Each balloon circles the continent from one to three times between launch and recovery. This opportunity is available for only 2-3 payloads per austral summer, so NASA is developing a Super Pressure Balloon (SPB) that would allow comparable durations at stable altitudes in mid-latitude regions for investigations that require nighttime exposure.

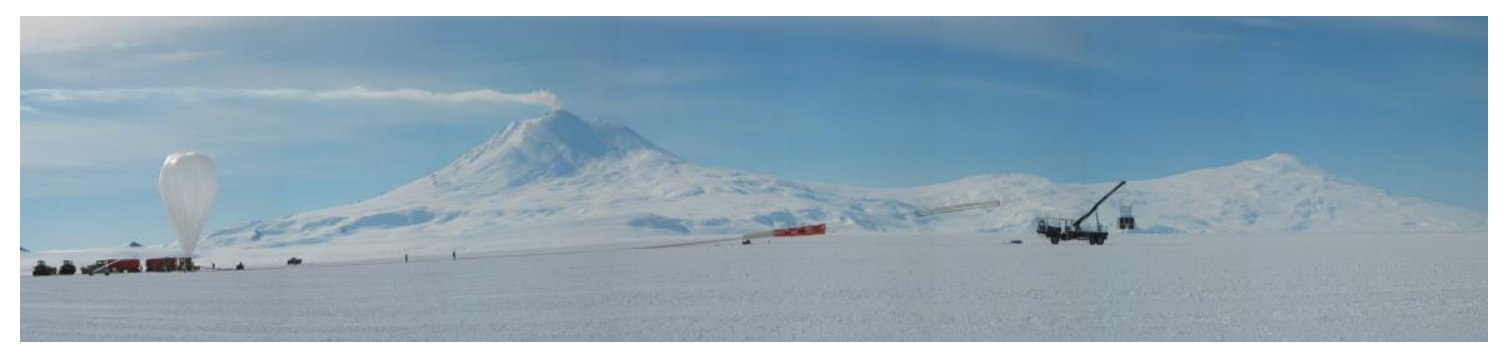

Fig. 1. View of pending balloon launch in Antarctica, with Mt. Erebus in the background.

Particle astrophysics has a ballooning history that spans over 100 years, ever since Victor Hess discovered cosmic rays on a manned balloon in 1912 [1]. The accumulations of discoveries made since then provide insight into the origin, acceleration and transport of Galactic cosmic rays [2,3]. Current particle astrophysics measurements include cosmic ray elemental abundances, isotopic composition, and energy spectra, as well as searches for neutrinos, antimatter, exotic particles, and dark matter candidates. In recent years it has also supported suborbital class investigations that utilize the ISS, which is especially attractive for investigations that study the low fluxes of high-energy cosmic rays.

The very successful Cosmic Ray Energetics and Mass (CREAM) investigation restructured its balloon-borne project budget to reconfigure one of its two proven payloads for exposure on the ISS, dubbed ISS-CREAM, pronounced "Ice" CREAM [4]. NASA has also selected a U.S. science team for participation in the Japanese-led Calorimetric Electron Telescope CALET [5]. The Alpha Magnetic Spectrometer (AMS) team sponsored by the U.S. Department of Energy was an early benefactor of the ISS [6]. Another U.S. science team was selected by NASA to participate in an international mission study for the Extreme Universe Space Observatory on the Japanese Experiment Module of the ISS, called (JEM-EUSO) [7]. The science objectives of these four complementary missions will be discussed further as a virtual package, dubbed Cosmic Ray Observatory on the ISS, in Sec. 5. 


\section{Vented Zero Pressure Balloons}

Vented zero-pressure (in equilibrium with the atmosphere) balloons have changed only incrementally from those introduced in the 1950's [8]. These large polyethylene balloons offer a unique capability for frequent access to near-space for science and technology instruments ranging in mass from a few kilograms $(\mathrm{kg})$ to $1000-2000 \mathrm{~kg}$. They traditionally carried payloads for 1 - 2 days, but in the early 1990's flight durations were extended to 10 - 20 days by conducting launches in the constant sunlight during local summers over Antarctica. These socalled Long-duration balloon (LDB) flights employ zero-pressure polyethylene balloons identical to those utilized for conventional non-polar flights, which are severely limited in duration by gas loss during day-night transitions.

The NASA-U.S. National Science Foundation Office of Polar Programs (NSF/OPP) partnership that established the Antarctic LDB program provided a sea change in scientific ballooning. Circumpolar LDB flights have been spectacularly successful, with many investigations utilizing multiple flights of payloads that are recovered, refurbished, and reused to minimize life-cycle costs [9]. The attainment of 25 - 32 day and 35 - 55 day flights, respectively, in two and three circumnavigations of Antarctica has greatly increased the expectations of scientific users. Current requests for participation in the NASA-NSF Antarctic LDB program exceed the capacity of two or three flights per annual campaign.

\section{Super Pressure Balloons}

Constant sunlight during local summer in Polar Regions allows zero-pressure balloons to maintain their float altitudes for long periods of time. However, mid-latitude flights using zeropressure balloons are limited to only a few days, because ballast must be dropped at each daynight transition to maintain float altitude. The volumes of zero-pressure balloons change as the ambient atmospheric pressure changes, causing a very large altitude droop at night. See Fig. 2. A SPB maintains nearly constant volume, thereby enabling long-duration flights in non-polar regions. These new balloons will carry sufficiently heavy payloads to high altitudes, so they will undoubtedly also bring another sea change in scientific ballooning by enabling mid-latitude LDB missions.
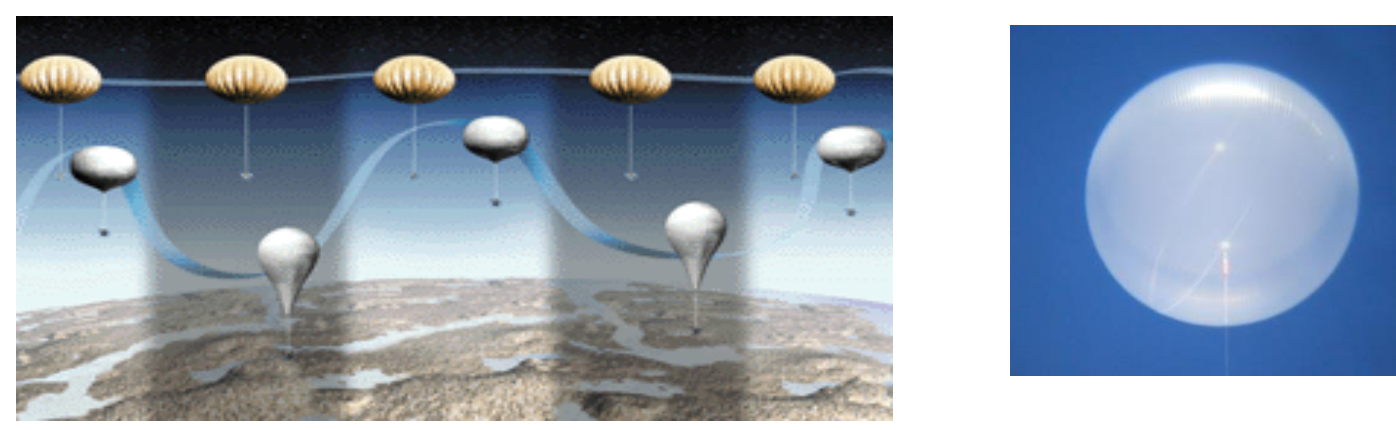

Fig. 2. (Left) Altitude stability for SPB (upper) and ZPB (lower) during day-night transitions; (Right) Photograph of SPB in its 110,000 ft float altitude.

The SPB is also key to the NASA balloon program's objective to establish an operational 60-100 day Ultra-Long Duration Balloon (ULDB) flight capability for the multidiscipline research community. Two qualification test flights of a 18.8 Million Cubic Foot (MCF) SPB balloon have been launched within the past year, one from near McMurdo, Antarctica and one from Wanaka, New Zealand, in order to certify an operational vehicle. The December 2014 SPB launch in Antarctica experienced a leak 
approximately 36 hours into the flight. This led to its termination over the Antarctica upper plateau, which precluded the plan to let it leave the continent for continued flight over the Southern Ocean, and thereby have a good chance to become the first ULDB test flight. Unfortunately, it also prevented retrieval of the balloon carcass for investigation of the anomaly.

The second test flight launched March 26, 2015 from Wanaka, NZ was flown successfully for 32 days and nights of level flight (the long way around the globe) at nearly constant altitude of 109,000 - 110,000 ft. before being terminated April 28, 2015 over Australia (Fig. 3). The balloon carcass was recovered along with the 5,000 lb. test payload, and both were returned to the NASA Balloon Program Office at the Wallops Flight Facility (WFF) for detailed study. NASA had earlier obtained permission to land the SPB and its payload in Argentina, Australia, or New Zealand, or in the ocean if necessary. This flight authorization would presumably apply also to future launches from Antarctica or Wanaka, NZ. The Compton Spectrometer and Imager (COSI) payload flown on the truncated December 2014 SPB flight in Antarctica is currently planned for launch from Wanaka in April 2016. And, plans are underway to conduct a followon SPB test flight with the EUSO Balloon payload one year later in April 2017.
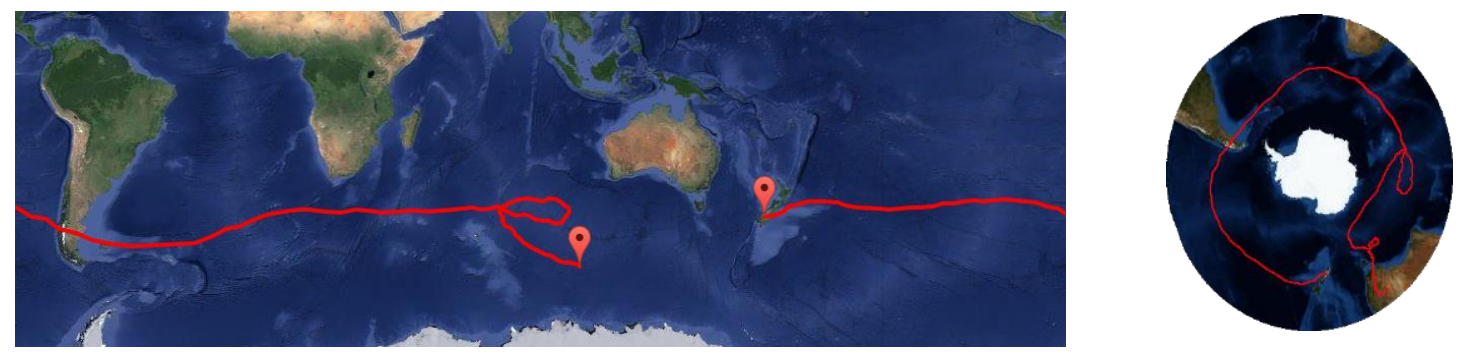

Fig. 3. (Left) Trajectory for first 18 days of the full 32-day SPB Trajectory (Right).

\section{Balloon-Borne Particle Astrophysics Investigations}

Antarctic LDB flights averaging about 17 days have supported various disciplines over the past two decades with important results. Recent results include the unexpected surplus of highenergy cosmic ray electrons reported by the Advanced Thin Ionization Calorimeter (ATIC), likely from a previously unidentified and relatively nearby cosmic object within about 1 kilo parsec of the Sun [10]. The Balloon-borne Experiment with a Superconducting Spectrometer (BESS) has conducted a negative search for annihilation signatures of dark matter in the antiproton channel [11]. The electron excess in ATIC and lack of excess antiprotons in BESS provide interesting constraints on dark matter models. A few of the key balloon-borne investigations of cosmic rays are highlighted below. The scope of this paper precludes many high priority balloon-borne investigations, and some within scope are not addressed because of space limitations, or because they are expected to be presented independently.

\subsection{Cosmic Ray Energetics and Mass (CREAM)}

CREAM was designed and built as a demonstration payload for NASA's ULDB vehicle [13]. Its goal was to extend direct measurements of cosmic-ray composition to the highest energies practical with LDB and ULDB flights over Antarctica. In six flights around Antarctica, this experiment accumulated 161 days of exposure, which is the longest duration ever achieved by a single balloon-borne project. It is equivalent to the original design goal for two nominal ULDB flights. The 42-day flight duration record that CREAM established in three circumnavigations of the continent during its first flight in the 2004 - 2005 austral season lasted for eight years, until it was broken by the Super-TIGER flight in the 2012 - 2013 season. It has made 
unprecedented spectral measurements of individual cosmic rays from hydrogen through iron over more than two decades in energy leading up to the "knee." The spectral hardening that it observed over these energies provide important constraints for propagation and acceleration models [14].

The rigidity dependence of the particle acceleration process in supernova leads to a characteristic change in elemental composition between the limiting energies for protons and iron, respectively about $\sim 10^{14}$ and $\sim 26 \times 10^{14} \mathrm{eV}$. This change is believed to be associated with a limit to supernova acceleration and the "knee" feature just above $10^{15} \mathrm{eV}$ in air shower data for the all-particle spectrum. The CREAM instrument is a quarter-scale version of the Advanced Cosmic-ray Composition Experiment for the Space Station (ACCESS) given high priority in the 2001 NRC Decadal study [15] to look for this characteristic signature. It meets the challenging and conflicting requirements for a large enough geometry factor to collect adequate statistics for the low flux of high-energy particles, and yet stay within the $1000 \mathrm{~kg}$ mass limit for ULDB flights..

\subsection{Super-Trans Iron Galactic Element Recorder (Super-TIGER)}

The very-large-area high-resolution Super-TIGER instrument measures the composition of galactic cosmic rays on high-altitude balloon flights over Antarctica. The record-breaking SuperTIGER-1 had a highly successful 55-day flight in the 2012 - 2013 austral season [15]. Its excellent data quality enables clear resolution of all $Z=10-40$ nuclei, and there appears to be clear resolution of elements in the $Z=40-60$ range, although statistics are low. The high performance of the instrument and the science that can be addressed by measuring $Z>40$ nuclei make a compelling case to conduct additional flights to measure the abundances of individual nuclei up to $Z=60$. The primary objective of SuperTIGER-2 is to measure for the first time the abundances of individual $41 \leq Z \leq 60$ nuclei, while substantially increasing the number of $30 \leq$ $\mathrm{Z} \leq 40$ nuclei obtained. These new measurements would provide sensitive tests and clarification of the OB-association model of galactic cosmic-ray origins, and they would test models for atomic processes by which nuclei are selected for acceleration to cosmic ray energies. They would also show whether the enrichment of refractory elements (those that exist primarily in dust grains in the interstellar medium) over volatile elements (those that exist primarily in the gas phase) extends into the $Z=41-60$ charge range.

\subsection{Antarctic Impulsive Transient Antenna (ANITA)}

NASA's only neutrino experiment was flown successfully for 35 days (ANITA-1), 33 days (ANITA-2), and 22 days (ANITA-3) during the 2006-2007, 2008-2009 and 2014-2015 Antarctic summers. This investigation offers a unique capability, not achievable with either ground-based instruments or instruments on spacecraft, of monitoring neutrinos at extreme energies above $\sim 10^{18} \mathrm{eV}$. It places an antenna array at the virtual "focus" of a neutrino ice telescope having an area of a million square kilometers on board a balloon flying at $\sim 40 \mathrm{~km}$ over the Antarctic continent [16]. The radio clarity of Antarctic ice, combined with the so-called Askaryan effect, enables a telescope with the dimensions needed to study cosmogenic neutrinos in an energy regime where they directly probe the nature of the highest energy particles in the universe. Although ANITA was designed to detect radio signals from the so-called Greisen-ZatsepinKuzman (GZK) neutrinos, it has turned out to be sensitive also to the highest energy air showers around and beyond this cutoff.

The IceCube observatory has found the first evidence for $\mathrm{PeV}$ cosmic neutrinos, so the scientific importance of probing any potential spectral tail of these neutrinos is high. ANITA can achieve adequate sensitivity to constrain the UHE neutrino spectrum, and ANITA-4 in combination with ANITA-3 will extend this sensitivity by an order of magnitude. The 
combination of ANITA-3 and ANITA-4 will make a compelling investigation of potential EeV neutrino fluxes, complementing the lower-energy IceCube measurements. The EVA mission builds on ANITA's successful approach by extending the sensitivity up to two orders of magnitude. This is done through a novel ultra-large radio antenna design embedded in the balloon system itself. It aims to develop a next-generation ultra-high energy astroparticle observatory capable of detection of neutrinos via the Askaryan effect in Antarctic ice, as well as charged cosmic-ray particles through the geomagnetic radiation of atmospheric extensive air showers.

\section{Cosmic Ray Observatory on the ISS}

A large fraction of the objectives in the list of Greatest Unanswered Questions of Physics [17] can be answered by particle astrophysics research. Many of them are identified in NASA's Strategic Goals [18] and Science Plan [19] developed from decadal surveys with community input. Each of the investigations, AMS [6], CALET [5], CREAM [4], and JEM-EUSO [7], addresses one or more of these unanswered questions, and all four of them are well suited for the ISS. Their collective science objectives validate the importance of the ISS for enabling a wide range of investigations crucial for understanding our universe and its fundamental nature.

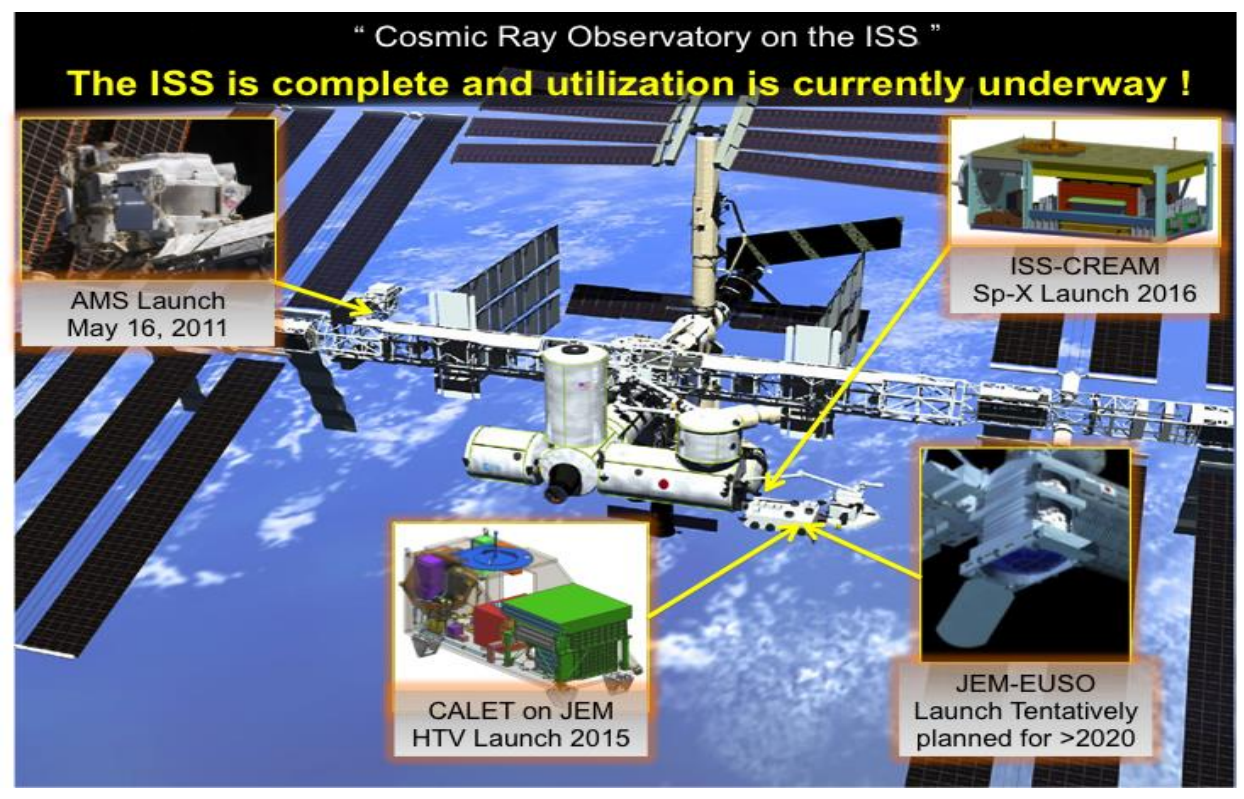

Fig. 4. Virtual "Cosmic Ray Observatory on the ISS."

\subsection{The Alpha Magnetic Spectrometer (AMS)}

The AMS instrument was launched by Space Shuttle Endeavor and installed on the ISS May 2011 to conduct a multi-messenger study of cosmic rays, including solar energetic particles and searches for dark matter and antimatter [6, 20]. Precise AMS measurements utilizing technology developed in particle physics and modified for space application should provide the means to confirm or refute much of the prior data [e.g., 21, 22]. Particles are distinguished from antiparticles by observing the oppositely curved tracks of particles with positive and negative charges traversing the strong magnetic field. 


\subsection{Calorimetric Electron Telescope (CALET)}

The CALET Mission to the ISS has been under development for the past decade as a Japanese led international mission involving Italy (CALET-IT) and the United Sates (CALET-US) $[5,23]$. It will extend measurements of the cosmic ray electron, nuclei and gamma-ray components into the trans-TeV energy region, beyond the energy reach of current experiments, to investigate possible new astrophysics at the highest energies. CALET is now ready for launch on HTV-5 in August 2015, having completed environmental testing (Acoustic, T/V, EMI/EMC) at the JAXA space center. The U.S. team has established the US CALET Data Center at Louisiana State University, acquired the hardware, developed the software, and verified connectivity with the Waseda Operations Center in Japan. The US Data Center will distribute data to the US investigators, process and analyze the CALET data stream to obtain science results, and prepare the CALET data products for archiving at the HEASRC facility at GSFC.

\subsection{Cosmic Ray Energetics and Mass for the ISS (ISS-CREAM)}

Building on the success of CREAM balloon flights, the instrument has been transformed for exposure on the ISS, which offers an order of magnitude increase in exposure and accumulated data [4. 9]. Its goal remains to extend direct measurements of cosmic ray composition to the highest energies practical, and have enough overlap with indirect ground-based measurements to answer illusive questions on cosmic ray origin, acceleration and propagation. Transforming the balloon instrument into ISS-CREAM has involved identification and replacement of components that might be at risk in the ISS environment, in addition to assessing safety and mission assurance concerns. This process has included rigorous testing of components to reduce risks and increase the likelihood of survivability on the launch vehicle and operations on the ISS without negatively impacting the heritage of the successful CREAM design. It has completed $\mathrm{EMI} / \mathrm{EMC}$, vibration, and acoustic tests, and it is now being prepared for thermal vacuum tests.

\subsection{Extreme Universe Space Observatory on the JEM-EF (JEM-EUSO)}

The JEM-EUSO instrument would use the Earth's atmosphere as a gigantic detector of a large number of extreme energy (>60 EeV) cosmic rays to increase the reach of fundamental physics probes to the most powerful accelerators in the universe [7, 24]. The US participants have focused on the Global Light System (GLS), a worldwide ground-based network of remotely operated light sources (lasers and Xe flashers) for onorbit calibration and monitoring. The GLS could be supplemented with an aircraft system. Calibrated UV lasers and Xenon flash lamps are capable of generating optical signatures with characteristics similar to extensive air showers initiated by cosmic rays within the instrument field of view. The JEM-EUSO mission should be able to reconstruct the pointing directions and energies of the lasers and flash lamps, in order to monitor the detector's triggers and accuracy of energy and direction reconstruction. A prototype of the JEM-EUSO instrument was flown on a balloon in 2014 to test its design.

\section{Conclusions}

There is much left to do to be ready for the next Decadal Study, but all balloon platforms (vented ZPB, SPB, and ULDB) and researchers will be vying for longer and longer flights, while supporting continued evolution of the ISS.

\section{Acknowledgements}

The author thanks the ISS Program Office, the Balloon Program Office, the Columbia Scientific Balloon Facility, and the many researchers, worldwide, who use of these platforms. 


\section{References}

[1] V. F. Hess, Z. Phys. 13 (1912) 1084.

[2] W.V. Jones, Advances in Space Research 53, (2014) 1405-1414.

[3] W.V. Jones, Brazilian J. Phys. Vol. 44/5, (2014) 534-539.

[4] E. S. Seo et al., 33 ${ }^{\text {rd }}$ Int. Cosmic Ray Conf., Rio De Janiero, Brazil (2013) ID 0629.

[5] S. Torii et al., 33 ${ }^{\text {rd }}$ Int. Cosmic Ray Conf., Rio De Janiero, Brazil (2013) ID 0245.

[6] M. Aguilar et al., Phys. Rep. 366-6 (2002) 331- 404.

[7] Y. Takahashi et al., New J. Phys. 11 (2009) 065009.

[8] W.V. Jones et al., Proc. 29th Int. Cosmic Ray Conf., Pune, India 10 (2005) 173-184.

[9] E. S. Seo, Astropart. Phys. 39/40 (2012) 76-87.

[10] J. Chang et al., Nature 456 (2008) 362-365.

[11] K. Abe, et al., Phys. Rev. Lett. 108/5 (2012) 051102.

[12] H. S. Ahn, et al., Nucl. Instrum. Methods A 579 (2007) 1034-1053.

[13] H. S. Ahn et al., Astrophys. J. Lett. 714 (2010) L89-L92.

[14] “Astronomy and Astrophysics in the New Millennium," National Academy Press, Washington, D.C., (2001).

[15] C. S. Powell, DiscoverMagazine.com/outthere, 06, 2013, 68-69.

[16] P. W. Gorham, et al., Phys. Rev. D. 82 (2010) 022004.

[17] “A 21st Century Frontier of Discovery: The Physics of the Universe”, A Report of the Interagency Working Group on the Physics of the Universe, National Science and Technology Council, Committee on Science (2004).

[18] "2006 NASA Strategic Plan, http://www.nasa.gov/about/budget/index.html, NP-2006-02-423-HQ.

[19] "Science Plan for NASA’s Science Mission Directorate 2007-2016," http://science.hq.nasa.gov/strategy/Science_Plan_07.pdf

[20] M. Aguilar et al., Phys. Rev. Lett. 110 (2013) 141102.

[21] O. Adriani et al., Nature 458 (2009) 607-609.

[22] A. A. Abdo et al., Phys. Rev. Lett. 102 (2009) 181101.

[23] M. Mori et al., 33 ${ }^{\text {rd }}$ Int. Cosmic Ray Conf., Rio De Janiero, Brazil (2013) ID 0248.

[24] G. Medina-Tanco et al., 33 ${ }^{\text {rd }}$ Int. Cosmic Ray Conf., Rio De Janiero, Brazil (2013) ID 0927. 